\title{
МЕЖПОКОЛЕННАЯ ТРАНСМИССИЯ ЦЕННОСТЕЙ В ГОРОДСКОЙ И СЕЛЬСКОЙ СРЕДЕ
}

\author{
Д.И. ДУБРОВ ${ }^{\mathrm{a}}$ А.Н. ТАТАРКО
}

${ }^{a}$ Национальный исследовательский университет «Высшая школа экономики», 101000, Россия, Москва, ул. Мясницкая, д. 20

\begin{abstract}
Резюме
Исследование посвящено рассмотрению трансмиссии индивидуальных ценностей, в частности влияния социально-культурного контекста проживания (городская и сельская среда) на сходство и различие ценностей подростков и их родителей. Методом исследования являлся социально-психологический опрос. В качестве инструментария использовался опросник индивидуальных ценностей Ш. Шварца (PVQ-R). В ходе исследования были опрошены представители двух поколений россиян: родители и подростки из 91 московской семьи и 62 семей в сельской местности (n = 306 человек). При математико-статистической обработке данных использовались: t-критерий Стьюдента, внутриклассовая корреляция и множественный дисперсионный анализ. В результате исследования установлено, что внутрисемейное сходство в выраженности ценностей, входящих в блоки Открытость изменениям, Самоутверждение и Самопреодоление, несколько выше в семьях, проживающих в городе, чем в семьях, проживающих в селе, а сходство ценностей блока Сохранение выше в семьях, проживающих в селе. Также установлено, что ценности подростков более сходны с ценностями сверстников, чем с ценностями родителей как в городском, так и в сельском социумах. Но при этом были выявлены ценностные различия между городскими и сельскими семьями. Наибольшие различия наблюдались по ценностному блоку Самопреодоление. В семьях, проживающих в городе, сходство по данному ценностному блоку выше, чем в семьях, проживающих в сельской местности. В обеих выборках наблюдались два варианта трансмиссии ценностей: от родителей к детям и влияние ценностей друзейсверстников, а также социально-культурного контекста (места проживания). В городском социуме лучше передаются ценности блока Самопреодоление, а в сельском - ценности блока Сохранение.
\end{abstract}

Ключевые слова: индивидуальные ценности, межпоколенная трансмиссия ценностей, сходства ценностей, социализация.

Статья подготовлена в результате проведения исследования в рамках Программы фундаментальных исследований Национального исследовательского университета «Высшая школа экономики» (НИУ ВШЭ) и с использованием средств субсидии в рамках государственной поддержки ведущих университетов Российской Федерации «5-100». 


\section{Основные теоретические подходы к исследованию межпоколенной трансмиссии ценностей}

В последние годы межпоколенная трансмиссия ценностей в семьях вызывает все больший интерес (Barni, 2009). В литературе часто можно встретить еще один термин, обозначающий данный феномен: культурная трансмиссия. Поскольку ценности напрямую связаны с культурой, то можно определить данные понятия как синонимы (Schwartz, 2014). Как отмечают зарубежные исследователи, культурная трансмиссия важна для преемственности в обществе, так как она поддерживает связь между членами разных поколений и позволяет сохранить знания о культуре и культурные традиции (Schönpflug, 2001; Trommsdorff, 2009). В настоящее время ввиду социальноэкономических изменений наблюдается ценностный разрыв между поколениями, а также гетерогенность в ценностных ориентациях людей в разных социально-культурных контекстах (например, город и село) (Fuligni, Zhang, 2004; Swader, 2013). Следовательно, полученные нами результаты будут полезны для родителей, а также для педагогов и психологов, работающих с семьями и детьми.

Проанализировав определения понятия «межпоколенная трансмиссия ценностей» (Barni, 2009; Schönpflug, 2001; Trommsdorff, 2009), мы пришли к выводу, что данное понятие можно определить как передачу ценностей одного поколения к другому в процессе социализации личности.
Преемственность ценностей между поколениями - важная задача социализации и является необходимой для функционирования общества (Fuligni, Zhang, 2004; Schönpflug, 2001). Сейчас трансмиссия ценностей определяется как интерактивный процесс; предполагается, что родители и дети выступают как субъектами, так и объектами в процессе социализации (Kuczynski, Navara, 2006; Roest et al., 2009). Поскольку трансмиссия имеет интерактивный характер, она, возможно, служит причиной как различий между поколениями, так и сходств между ними. В литературе данный вариант трансмиссии ценностей называется вертикальным, это передача ценностей от родителей к детям (Berry et al., 1992; Cavalli-Sforza, Feldman, 1981).

Д. Барни установила, что, по мнению подростков, родители стремятся поддерживать в них в первую очередь консервативные ценности, в то время как они более открыты новому опыту и вызовам, поэтому подростки отказываются принимать ценности блока Сохранение (Barni, 2009).

Подростки исследуют и развивают свою автономию и независимую идентичность. Ценности блока Открытость изменениям, способствующие достижению независимости, являются наиболее эффективными при такой мотивации. С другой стороны, на этой стадии жизненного цикла родители должны ответственно заботиться о своих детях. Родители должны учитывать и аспекты зависимости, и потребность в безопасности и правилах, которые продолжают действовать на протяжении подросткового возраста, точно так же, как и возрастающую 
потребность подростка в автономии (Там же).

Кроме родителей в процессе трансмиссии ценностей участвуют сами дети и их друзья-сверстники. Данный вариант трансмиссии в литературе называют «горизонтальным», т.е. на ценностные ориентации детей влияют друзья-сверстники (Berry et al., 1992; Cavalli-Sforza, Feldman, 1981). Можно сделать вывод о том, что в подростковом возрасте наиболее значимы ценностные влияния сверстников, чем родителей.

\section{Влияние среды проживания на трансмиссию ценностей}

Кроме описанных влияний (члены семьи, сверстники, друзья и их взаимоотношения) на ценности родителей и подростков влияет социально-культурный контекст, в котором данные отношения протекают (Trommsdorff, 2009). Возникает вопрос: меняются ли процесс трансмиссии ценностей и его результат от поколения к поколению в зависимости от социального контекста (тип населенного пункта - город или село и т.д.)?

Ответ на данный вопрос попытались дать А. Фулиньи и У. Цанг через исследование отношения подростков, проживающих в городском и сельском социуме современного Китая, к семейным обязанностям. Китай сравнительно недавно перешел на рыночную экономику, поэтому задачей авторов исследования было выяснить, какие изменения произошли в ценностях подрастающего поколения и как на это влияет место проживания.
Были получены следующие результаты: позитивное отношение к семейным обязанностям менее характерно для подростков мужского пола, проживающих в городе. Данный факт может быть объяснен тем, что в городе строго регулируется рождаемость, поэтому практически все респонденты были единственными детьми в семьях, а это, по мнению ряда исследователей, способствует развитию индивидуализма. Также в сельской местности менее развита система социального обеспечения, поэтому всем членам семьи приходится больше времени уделять заботе о близких. Кроме того, было выявлено, что данный аттитюд проявлялся сильнее в семьях, где преобладают близкие, теплые, доверительные отношения. Авторы предполагают, что данный фактор является универсальным для всех стран (Fuligni, Zhang, 2004).

Таким образом, в целом семья имеет большое значение для респондентов, поэтому они принимают и выполняют семейные обязанности. Проанализировав данные психологических исследований (Fuligni, Zhang, 2004; Barni, 2009; Жарова, 2010; Виноградский, 1998; Фадеева, 2007; Мудрик, 2000), мы пришли к выводу, что социальный контекст (город - село) влияет на ценности подростков и их родителей. На основании данного вывода мы сформулировали гипотезу о том, что сходство в ценностных блоках Открытость изменениям и Самоутверждение выше в семьях, проживающих в городе, чем в семьях, проживающих в селе, а сходство в блоках Сохранение и Самопреодоление выше в семьях, проживающих в селе. 
Основной целью проведенного исследования является эмпирическая проверка влияния социального контекста на сходства и различия ценностей родителей и подростков в городской и сельской среде.

\section{Гипотезы исследования}

Н1. Ценности подростков в городском социуме более сходны с ценностями сверстников, чем родителей.

Н2. Сходство ценностей подростков и их родителей в ценностных блоках Открытость изменениям и Самоутверждение выше в семьях, проживающих в городе, чем в семьях, проживающих в селе, а сходство ценностей подростков и их родителей в блоках Сохранение и Самопреодоление выше в семьях, проживающих в селе.

\section{Процедура и методика исследования}

\section{Участники}

Для определения сходств и различий ценностей подростков и родите- лей в разном социальном контексте мы опросили представителей двух поколений (родители, дети-подростки), проживающих в Москве и селах Ярославской, Тверской и Ивановской областей.

В таблице 1 представлены гендерные и возрастные характеристики выборок.

\section{Процедура}

Исследование проводилось на базе школ города Москвы и сельских поселений Ярославской, Ивановской, Тверской областей. Подросткам раздавались анкеты, которые они заполняли либо в школе, либо дома. Также подросткам были даны анкеты, которые необходимо было заполнить одному из родителей.

\section{Инструментарий}

Эмпирическое исследование сходств и различий ценностей родителей и подростков в городской и сельской среде осуществлялось с помощью метода социально-психологического опроса - анкетирования.

Демографические характеристики выборок исследования

\begin{tabular}{|l|c|c|c|c|}
\hline \multicolumn{7}{|c|}{ Общее количество } & \% мужчин & \% женщин & М (SD) возраст \\
\hline \multicolumn{7}{|c|}{ Семьи, проживающие в городе } \\
\hline Подростки & 90 & $40 \%$ & $60 \%$ & $17.6(1.71)$ \\
\hline Родители & 90 & $22.2 \%$ & $77.8 \%$ & $43.3(5.71)$ \\
\hline \multicolumn{7}{|c|}{ Семьи, проживающие в селе } \\
\hline Подростки & 62 & $53.2 \%$ & $46.8 \%$ & $15.6(1.75)$ \\
\hline Родители & 62 & $21 \%$ & $79 \%$ & $41.7(5.39)$ \\
\hline Всего & 304 & $33.8 \%$ & $66.2 \%$ & \\
\hline
\end{tabular}


Для данного исследования был использован опросник индивидуальных ценностей Ш. Шварца PVQ-R (Шварц и др., 2012).

Проверка гипотез осуществлялась с помощью следующих методов математической статистики, реализованных в программе SPSS: множественный дисперсионный анализ, t-критерий Стьюдента.

\section{Описание полученных результатов}

Проверка первой гипотезы осуществлялась с помощью подсчета ценностных профилей городских и сельских подростков, внутриклассовых корреляций между ценностями подростков и их родителями в каждой семье и между ценностями подростков и ценностными профилями сверстников, а затем использовались множественный дисперсионный анализ и t-критерий Стьюдента для определения значимости различий.

Результаты представлены в таблицах 2 и 3.

Как видим, внутри выборок и в селе и в городе профиль ценностей подростков ближе к профилю ценностей сверстников, чем к профилю ценностей родителей. Но в целом размеры внутриклассовых коэффициентов корреляций статистически значимо не различаются у респондентов из города и из села.

Теперь посмотрим, различаются ли городская и сельская выборки по данным показателям. Для этого снова используем множественный дисперсионный анализ. Получились следующие данные: $\lambda$ Уилкса $=.994$; $\mathrm{F}(2,149.000)=.435 ; p=.648 ; \eta^{2}=.006$.

Как видим, городская и сельская выборки не различаются по данным показателям.

Ценности подростков более сходны с ценностями сверстников, чем с ценностями родителей, как в городском, так и в сельском социуме.

Проверка второй гипотезы осуществлялась путем подсчета внутриклассовой корреляции между ценностями подростков и их родителей в каждой семье, далее проводился множественный дисперсионный анализ (MANOVA).

Зависимыми переменными в исследовании выступали ценностные блоки. Независимой переменной явилось место проживания (город - село). Получились следующие значения: $\lambda$ Уилкса $=.931 ; \mathrm{F}$ (4, $147.000)=2.73 ; p=0.031 ; \eta^{2}=0.069$. Как мы видим, в показателях по ценностным блокам имеются значимые различия в зависимости от места проживания (город - село). Данные представлены в таблице 4.

Таблица 2

Сравнение сходств ценностей подростков и родителей в городе и селе (t-критерий Стьюдента)

\begin{tabular}{|l|c|c|c|c|c|c|c|}
\hline \multirow{2}{*}{$\begin{array}{c}\text { Профили ценностей } \\
\text { (город/село) }\end{array}$} & \multicolumn{2}{|c|}{ Город $(\mathrm{N}=90)$} & \multicolumn{2}{|c|}{ Село $(\mathrm{N}=62)$} & \multirow{2}{*}{$t$} & \multirow{2}{*}{$d f$} & \multirow{2}{*}{$p$} \\
\cline { 1 - 6 } & $\mathrm{M}$ & $\mathrm{SD}$ & $\mathrm{M}$ & $\mathrm{SD}$ & & & \\
\hline Подростки-родители & .365 & .28 & .340 & .32 & 0.51 & 117.09 & .62 \\
\hline Подростки-сверстники & .527 & .14 & .499 & .23 & 0.93 & 94.56 & .36 \\
\hline
\end{tabular}


Сравнение сходств ценностей подростков и родителей в городе и селе (множественный дисперсионный анализ)

\begin{tabular}{|l|c|c|c|c|}
\hline \multicolumn{1}{|c|}{ Зависимая переменная } & Город $\mathbf{M ( r )}$ & Село $\mathbf{M ( r )}$ & $\mathbf{F}$ & $\boldsymbol{\eta}^{\mathbf{2}}$ \\
\hline Подростки-родители & .366 & .340 & 0.271 & .002 \\
\hline Подростки-сверстники & .528 & .499 & 0.859 & .006 \\
\hline
\end{tabular}

Примечание. M(r) - среднее значение внутриклассовой корреляции.

Сравнение сходств ценностей подростков и родителей в городе и селе

\begin{tabular}{|l|c|c|c|c|}
\hline \multicolumn{1}{|c|}{ Ценностный блок } & Город $\mathbf{M ( r )}$ & $\mathbf{C е л о ~} \mathbf{M ( r )}$ & $\mathbf{F}$ & $\boldsymbol{\eta}^{\mathbf{2}}$ \\
\hline Сохранение & .301 & .325 & 0.122 & .001 \\
\hline Открытость изменениям & .226 & .126 & 1.502 & .010 \\
\hline Самоутверждение & .459 & .436 & 0.106 & .001 \\
\hline Самопреодоление & .528 & .359 & $9.247^{*}$ & .058 \\
\hline
\end{tabular}

$* p<0.05$.

Статистически значимый вклад в данные различия вносит только ценностный блок Самопреодоление. По ценностному блоку Самопреодоление большее сходство среди подростков и их родителей наблюдается в городской выборке.

Больше статистически значимых различий по блокам ценностей не обнаружено.

\section{Обсуждение результатов эмпирического исследования}

Итак, согласно полученным данным, гипотеза 1 подтвердилась, гипотеза 2 подтвердились частично, поскольку сходство по блоку Cамопреодоление значимо выше в семьях, проживающих в городе, чем в селе. Данный факт можно объяснить тем, что в состав ценностного блока Самопреодление входят ценности
Универсализм - Толерантность и Универсализм - Забота о природе. Именно по данным ценностям в городской выборке наблюдаются наибольшие значения.

Как мы уже отмечали, в сельской местности уровень социального контроля выше, чем в городе (Жарова, 2010), поэтому не одобряются какие-либо отклонения от общепринятых норм, следовательно, в селе по данной ценности средние значения ниже, чем в городе. Этим же можно объяснить более высокий уровень значений и сходств по ценностному блоку Сохранение (Конформизм, Традищия, Безопасность) в селе.

Что касается ценности Универсализм - Забота о природе, то здесь, на наш взгляд, играет роль экологический аспект. Люди, проживающие в городах, уже столкнулись с проблемами экологии, поэтому забота о 
природе перешла в разряд «ценности внешнего статуса» - наиболее значимой (Ядов, 1975), а для сельской местности проблемы экологии менее актуальны, поэтому забота о природе для сельских жителей, согласно ядерно-иерархической модели ценностей, является ценностью среднего или ниже среднего статуса, т.е. менее значимой (Там же). Данную ценность родители, проживающие в селе, не стремятся передавать своим детям.

Как видим, в данном случае наблюдается «вертикальный» вариант трансмиссии ценностей, т.е. от родителей к детям.

Кроме этого, данные, полученные на городской выборке, частично объясняют и подтверждают данные, полученные Д. Барни и ее коллегами (Barni et al., 2011). В исследовании Барни наибольшее расхождение в ценностных ориентациях между подростками и их родителями наблюдалось по блокам Открытость изменениям и Сохранение. Барни указывала на то, что, по мнению подростков, родители стремятся поддерживать в них в первую очередь ценности блока Сохранение (Традищия, Безопасность, Конформизм), в то время как они более открыты новому опыту и вызовам. Подростки отказываются принимать данные ценности, исследуют и развивают свою автономию и независимую идентичность. Ценностный блок Открытость изменениям, способствующий достижению независимости, является наиболее эффективным при данной мотивации (Barni, 2009).

Влияние социального контекста (место проживания) на данный процесс подтверждают не только полу- ченные нами данные, но и результаты других исследований (Fuligni, Zhang, 2004; Жарова, 2010).

Отсутствие значимых различий между значениями внутриклассовой корреляции ценностей семей и сверстников, проживающих в городе и селе, можно объяснить наличием общего ценностного климата, преобладающего в обществе «духа времени» (zeitgeist), на который в своих исследованиях указывали Клаус Бонке и другие исследователи (Boehnke, 2001; Boehnke et al., 2007; Vedder et al., 2009).

Тот же эффект сработал и на выборке подростков, проживающих в городе. Их ценности оказались более сходными с ценностями их сверстников, нежели с ценностями родителей.

Таким образом, полученные нами данные во многом соотносятся с результатами зарубежных исследований (Barni, 2009; Steca et al., 2012; Fuligni, Zhang, 2004). Можно утверждать, что социально-культурный контекст проживания влияет на процесс межпоколенной трансмиссии ценностей.

\section{Выводы}

1. Уровень сходства ценностей подростков и их родителей по четырем ценностным блокам (Сохранение, Открытость изменениям, Самоутверждение и Самопреодоление) различается в городе и селе. Наблюдается влияние социально-культурного контекста (город - село) на трансмиссию ценностей.

2. Сходство в ценностных блоках Открытость изменениям, Самоутверждение и Самопреодоление несколько выше в семьях, проживающих в городе, чем в семьях, прожи- 
вающих в селе. Эти блоки ценностей транслируются от родителей к детям преимущественно в городском контексте. Сходство в ценностях блока Сохранение выше в семьях, проживающих в селе, т.е. эти ценности преимущественно транслируются от родителей к детям в более традиционном сельском контексте.

3. По всем ценностным блокам ценности подростков более сходны с ценностями сверстников, чем родителей, как в городском, так и в сельском контекстах. В данном случае наблюдается вариант «горизонтальной» трансмиссии, когда на ценности подростков влияют ценности их друзей-сверстников. Для подростков более значимы ценности сверстников, чем родителей.

4. Обобщая результаты исследования, можно сказать, что оба известных варианта трансмиссии ценностей («горизонтальная» и «вертикальная») наблюдаются и в городском, и сельском контекстах. Но при «вертикальной» трансмиссии не все ценности передаются от родителей к детям. В городском контексте от родителей к детям преимущественно транслируются ценности блока Самопреодоление, а в сельском - ценности блока Сохранение. «Горизонтальная» трансмиссия (от подростков к подросткам) является одинаковой по всем четырем блокам ценностей.

\section{Литература}

Виноградский, В. Г. (1998). Крестьянские семейные хроники. Сочиологический журнал, 1(2), $130-144$.

Жарова, Е. Н. (2010). Сравнительный анализ индивидуалистических и коллективистических ценностных ориентаций как сочиально-психологических характеристик молодежи (на примере урбанизированной и неурбанизированной среды) (Автореферат кандидатской диссертации, МГППУ, Москва).

Мудрик, А. В. (2000). Основные составляющие влияния мезофакторов на социализацию человека. В кн. В. А. Сластенин (Ред.), Социальная педагогика (с. 31-50). М.: Издательский центр «Академия».

Фадеева, О. П. (2007). Хозяйственные уклады в современном российском селе. Социологические исследования, 11, 64-69.

Шварц, Ш., Бутенко, Т. П., Седова, Д. С., Липатова, А. С. (2012). Уточненная теория базовых индивидуальных ценностей: применение в России. Психология. Журнал Высшей школь экономики, 9(2), 43-70.

Ядов, В. А. (1975). О диспозиционной регуляции социального поведения личности. В кн. Методологические проблемы социальной психологии (с. 89-106). М.: Наука.

Barni, D. (2009). Trasmettere valori. Tre generazioni familiari a confronto [Transmittig values. A comparison between three family generations]. Milano: Unicopli. (in Italian)

Barni, D., Ranieri, S., Scabini, E., \& Rosnatiet, R. (2011). Value transmission in the family: do adolescents accept the values their parents want to transmit? Journal of Moral Education, 1(40), 105-121.

Berry, J. W., Poortinga, Y. H., Segall, M. H., \& Dasen, P. R. (1992). Cross-cultural psychology: Research and applications. New York: Cambridge University Press.

Boehnke, K. (2001). Parent-offspring value transmission in a societal context: Suggestions for a utopian research design with empirical underpinnings. Journal of Cross-Cultural Psychology, 32, 241-255. 
Boehnke, K., Hadjar, A., \& Baier, D. (2007). Parent-child value similarity: The role of Zeitgeist. Journal of Marriage and Family, 69, 778-792.

Cavalli-Sforza, L. L., \& Feldman, M. W. (1981). Cultural transmission and evolution: A quantitative approach. Princeton: Princeton University Press.

Fuligni, A., \& Zhang, W. (2004). Attitudes toward family obligations among adolescents in contemporary urban and rural China. Child Development, 74, 180-192.

Kuczynski, L., \& Navara, G. S. (2006). Sources of innovation and change in socialization, internalization and acculturation. In M. Killen \& J. G. Smetana (Eds.), Handbook of moral development (pp. 299-327). Mahwah, NJ: Lawrence Erlbaum Associates.

Roest, A. M. C., Dubas, J. S., \& Gerris, J. R. M. (2009). Value transmissions between parents and children: gender and developmental phase as transmission belts. Journal of Adolescence, 33(1), 21-31.

Schönpflug, U. (2001). Intergenerational transmission of values: The role of transmission belts. Journal of Cross-Cultural Psychology, 32, 174-185.

Schwartz, S. H. (2014). National culture as value orientations: Consequences of value differences and cultural Distance. In V. A. Ginsburgh \& D. Throsby (Eds.), Handbook of the economics of art and culture (Vol. 2, pp. 547-586). Amsterdam: Elsevier.

Steca, P., Monzan, D., Greco, A., \& D’Addario, M. (2012). Similarity in self-enhancement and self-transcendence values between young adults and their parents and friends. Family Science, 1(3), 34-45.

Swader, C. S. (2013). The capitalist personality: Face-to-face sociality and economic change in the postcommunist world. New York: Routledge.

Trommsdorff, G. (2009). Intergenerational relations and cultural transmission. In U. Schönpflug (Ed.), Cultural transmission. Psychological, developmental, social, and methodological aspects (pp. 126-160). New York: Cambridge University Press.

Vedder, P., Berry, J., Sabatier, C., \& Sam, D. (2009). The intergenerational transmission of values in national and immigrant families: The role of Zeitgeist. Youth Adolescence, 38, 642-653.
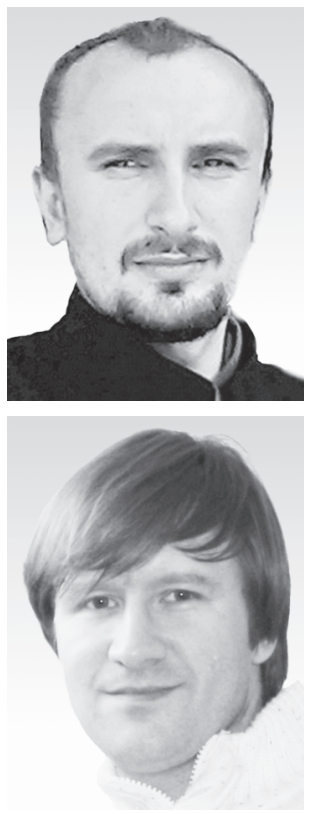

Дубров Дмитрий Игоревич - аспирант, департамент психологии, факультет социальных наук, и стажер-исследователь, Международная научно-учебная лаборатория социокультурных исследований, Экспертный институт, Национальный исследовательский университет «Высшая школа экономики».

Контакты: ddubrov@hse.ru

Татарко Александр Николаевич - доцент, департамент психологии, факультет социальных наук, и ведущий научный сотрудник, Международная научно-учебная лаборатория социокультурных исследований, Экспертный институт, Национальный исследовательский университет «Высшая школа экономики», доктор психологических наук.

Контакты: tatarko@yandex.ru 


\title{
Intergenerational Value Transmission in Urban and Rural Area
}

\author{
Dmitry I. Dubrov ${ }^{\mathrm{a}}$, Aleksandr N. Tatarko ${ }^{\mathrm{a}}$ \\ ${ }^{a}$ National Research University Higher School of Economics, 20 Myasnitskaya str., Moscow, 101000, \\ Russian Federation
}

\begin{abstract}
The research is devoted to transmission of individual values, in particular the impact of socio-cultural context of residence (urban and rural area) on the similarities and differences in values of adolescents and their parents. The research method was a socio-psychological survey. As the instrument we used the Portrait Values Questionnaire by S. Schwartz (PVQ-R). We interviewed representatives of two generations: parents and children from 91 families in Moscow and 62 families in rural areas $(n=306)$. For mathematical-statistical data processing we used: Student's ttest, intraclass correlation and multivariate analysis of variance. The study established that intra-family similarity in values from blocks Openness to Change, Self-Enhancement and Self-Transcendence is somewhat higher in the families living in the city than in the families living in the village, and the value similarity in block Conservation is higher in families residing in the village. It is also established that adolescents' values are more similar to the values of peers than to parents ' values, in both urban and rural societies. But at the same time, we identified value differences between urban and rural families. The greatest differences were observed in values of block Self-Transcendence. The value similarity of this block is higher in families living in the city than in families living in rural areas. In both samples we observed two options for the transmission of values: from parents to children and value influence of friends, peers, and socio-cultural context (place of residence). In urban society Self-Transcendence values are better transmitted, and in rural society Conservation values are better transmitted.
\end{abstract}

Keywords: individual values, intergenerational transmission of values, value similarity, socialization.

\section{References}

Barni, D. (2009). Trasmettere valori. Tre generazioni familiari a confronto [Transmittig values. A comparison between three family generations]. Milano: Unicopli. (in Italian)

Barni, D., Ranieri, S., Scabini, E., \& Rosnatiet, R. (2011). Value transmission in the family: do adolescents accept the values their parents want to transmit? Journal of Moral Education, 1(40), 105-121.

Berry, J. W., Poortinga, Y. H., Segall, M. H., \& Dasen, P. R. (1992). Cross-cultural psychology: Research and applications. New York: Cambridge University Press.

Boehnke, K. (2001). Parent-offspring value transmission in a societal context: Suggestions for a utopian research design with empirical underpinnings. Journal of Cross-Cultural Psychology, 32, 241-255.

Boehnke, K., Hadjar, A., \& Baier, D. (2007). Parent-child value similarity: The role of Zeitgeist. Journal of Marriage and Family, 69, 778-792.

Cavalli-Sforza, L. L., \& Feldman, M. W. (1981). Cultural transmission and evolution: A quantitative approach. Princeton: Princeton University Press.

Fadeeva, O. P. (2007). Khozyaistvennye uklady v sovremennom rossiiskom sele [Economic set-ups in the contemporary Russian village]. Sotsiologicheskie Issledovaniya, 11, 64-69. 
Fuligni, A., \& Zhang, W. (2004). Attitudes toward family obligations among adolescents in contemporary urban and rural China. Child Development, 74, 180-192.

Kuczynski, L., \& Navara, G. S. (2006) Sources of innovation and change in socialization, internalization and acculturation. In M. Killen \& J. G. Smetana (Eds.), Handbook of moral development (pp. 299-327). Mahwah, NJ: Lawrence Erlbaum Associates.

Mudrik, A. V. (2000). Osnovnye sostavlyayushchie vliyaniya mezofaktorov na sotsializatsiyu cheloveka [The main components of mesofactors influence on socialization of man]. In V. A. Slastenin (Ed.), Sotsial'naya pedagogika [Social pedagogy] (pp. 31-50). Moscow: Akademiya.

Roest, A. M. C., Dubas, J. S., \& Gerris, J. R. M. (2009). Value transmissions between parents and children: gender and developmental phase as transmission belts. Journal of Adolescence, 33(1), 21-31.

Schönpflug, U. (2001). Intergenerational transmission of values: The role of transmission belts. Journal of Cross-Cultural Psychology, 32, 174-185.

Schwartz, S. H. (2014). National culture as value orientations: Consequences of value differences and cultural Distance. In V. A. Ginsburgh \& D. Throsby (Eds.), Handbook of the economics of art and culture (Vol. 2, pp. 547-586). Amsterdam: Elsevier.

Schwartz, S., Butenko, T. P., Sedova D. S., \& Lipatova, A. S. (2012). A refined theory of basic personal values: validation in Russia. Psychology. Journal of Higher School of Economics, 9(2), 43-70.

Steca, P., Monzan, D., Greco, A., \& D’Addario, M. (2012). Similarity in self-enhancement and self-transcendence values between young adults and their parents and friends. Family Science, 1(3), 34-45.

Swader, C. S. (2013). The capitalist personality: Face-to-face sociality and economic change in the postcommunist world. New York: Routledge.

Trommsdorff, G. (2009). Intergenerational relations and cultural transmission. In U. Schönpflug (Ed.), Cultural transmission. Psychological, developmental, social, and methodological aspects (pp. 126-160). New York: Cambridge University Press.

Vedder, P., Berry, J., Sabatier, C., \& Sam, D. (2009). The intergenerational transmission of values in national and immigrant families: The role of Zeitgeist. Youth Adolescence, 38, 642-653.

Vinogradskii, V. G. (1998). Krest'yanskie semeinye khroniki [Peasant family chronicles]. Sotsiologicheskii Zhurnal, 1(2), 130-144.

Yadov, V. A. (1975). O dispozitsionnoi regulyatsii sotsial'nogo povedeniya lichnosti [On dispositional regulation of social behavior of personality]. In Metodologicheskie problemy sotsial'noi psikhologii [Methodological problems of social psychology ] (pp. 89-106). Moscow: Nauka.

Zharova, E. N. (2010). Sravnitel'nyi analiz individualisticheskikh i kollektivisticheskikh tsennostnykh orientatsii kak sotsial'no-psikhologicheskikh kharakteristik molodezhi (na primere urbanizirovannoi $i$ neurbanizirovannoi sredy) [Comparative analysis of individualistic and collectivistic value orientations as socio-psychological characteristics of young people (in urban and rural area)] (Extended abstract of Ph.D. dissertation, Moscow State University of Psychology \& Education, Moscow).

Dmitry I. Dubrov - postgraduate student, School of Psychology, Faculty of Social Sciences, intern researcher, International Scientific-Educational laboratory for Socio-Cultural Research, Expert Institute, National Research University Higher School of Economics.

E-mail: ddubrov@hse.ru

Alexander N. Tatarko - associate professor, Faculty of Social Sciences, School of Psychology, senior researcher, International Laboratory for Socio-Cultural Research, National Research University Higher School of Economics, D.Sc.

E-mail: tatarko@yandex.ru 